\title{
Parietal Area 5 and the Initiation of Self-Timed Movements versus Simple Reactions
}

\author{
Gaby Maimon and John A. Assad \\ Department of Neurobiology, Harvard Medical School, Boston, Massachusetts 02115
}

The timing of action has been studied extensively in reaction-time tasks in which an abrupt sensory stimulus triggers a movement. In these experiments, neurophysiologists have attempted to explain variability in movement time with variability in neuronal activity. However, in natural settings, movements are not usually triggered by abrupt sensory cues. What underlies the timing of action under such circumstances, when movements are uncoupled or only weakly coupled to abrupt events in the external world? We trained monkeys to perform the same arm movement either in direct reaction to a salient visual event, or as a self-timed action, less coupled to any obvious external trigger. Neurons in cortical area 5 exhibited phasic discharge modulations that were generally comparable for both modes of action, with some neurons increasing and others decreasing their firing rates with movement. For self-timed movements, however, there was an additional, slow ramp-up or ramp-down of activity in the few hundred milliseconds before the phasic discharge. These ramping modulations occurred well before any detectable changes in arm-muscle activity and their time course bore a striking resemblance to activity in the putamen preceding self-timed movements, observed previously. Together, the results suggest a possible mechanism for the internal timing of action within the motor system. In this model, reverberant activity in corticobasal-ganglia circuits reaches a threshold level resulting in much larger perimovement discharges within the same network, consequently driving the initiation of action.

Key words: area 5; parietal cortex; motor system; movement initiation; neurophysiology; macaque

\section{Introduction}

Some movements, like flinching, are direct, reflex-like reactions to external events. In these sorts of behaviors, a linear chain of causality seems clear: sensory responses time-locked to the stimulus could propagate through various stages of processing and eventually influence motor neurons. Variability in behavioral reaction times could arise from variability in sensory responses (Cook and Maunsell, 2002), premotor activity (Hanes and Schall, 1996; Gold and Shadlen, 2000), or in the fidelity of transmitting the trigger across processing stages (DiCarlo and Maunsell, 2004). Most natural movements, however, are not directly caused by external trigger events and, in these cases, it is less obvious how neuronal circuits determine the precise moment to initiate action. One could always ask: why not a second earlier or later?

Insight into this issue has come from examining neuronal activity preceding arm movements in monkeys. In these studies, the animals are required to make the same movement either as an immediate reaction to an external sensory cue, or as a selfgenerated movement, where action does not immediately follow any external stimulus. Single-cell recordings from thalamus, basal ganglia, and frontal-motor areas indicate that, although

Received Aug. 24, 2005; revised Dec. 26, 2005; accepted Jan. 17, 2006.

This work was supported by National Institute of Neurological Disorders and Stroke Grant NS-41000 and a National Science Foundation predoctoral fellowship to G.M. We thank Ron Maimon for helpful comments on this manuscript. Kimberly Irwin, Tim LaFratta, Mike LaFratta, John LeBlanc, and David Averbuch provided excellent technical assistance.

Correspondence should be addressed to John A. Assad, Department of Neurobiology, Harvard Medical School, Boston, MA 02115. E-mail: jassad@hms.harvard.edu.

DOI:10.1523/JNEUROSCI.3590-05.2006

Copyright $\odot 2006$ Society for Neuroscience $\quad$ 0270-6474/06/262487-12\$15.00/0 many movement-responsive neurons fire differently in the two conditions, all these regions are generally active during both modes of action (Okano and Tanji, 1987; Mink and Thach, 1991; Romo et al., 1992; Romo and Schultz, 1992; Schultz and Romo, 1992; van Donkelaar et al., 1999).

Lee and Assad (2003) introduced a novel behavioral paradigm that allowed for a reliable, controlled comparison between selfinitiated and reactive movements. In their task, a monkey received a start-of-trial cue, waited $2000 \mathrm{~ms}$, and then was free to move. If the animal moved with no further change to the visual stimulus, the movement was deemed self-timed. However, in some trials, before the animal moved on his own, a cue appeared on the screen requiring him to act within a reaction-time window. These movements were deemed cued. Recording in the sensorimotor putamen, Lee and Assad found that activity of handmovement responsive neurons was actually remarkably similar for all movements, except for one consistent difference: neurons exhibited slightly elevated firing rates a few hundred milliseconds before self-timed actions as compared with cued actions.

This selective neuronal activation for self-timed movements likely also involves frontal cortical areas (Romo and Schultz, 1992), but could it engage an even broader brain circuit? In particular, it has been hypothesized that parietal cortex plays a role in internal "command" of action (Mountcastle et al., 1975), and field potentials in parietal area 5 can precede movement onset by hundreds of milliseconds for self-paced movements (Gemba et al., 2004). Here, we trained monkeys to initiate arm movements either in direct reaction to a salient visual event or without an obvious external trigger. We found that many motor-related neurons in area 5 exhibited premovement activity that was selec- 
A

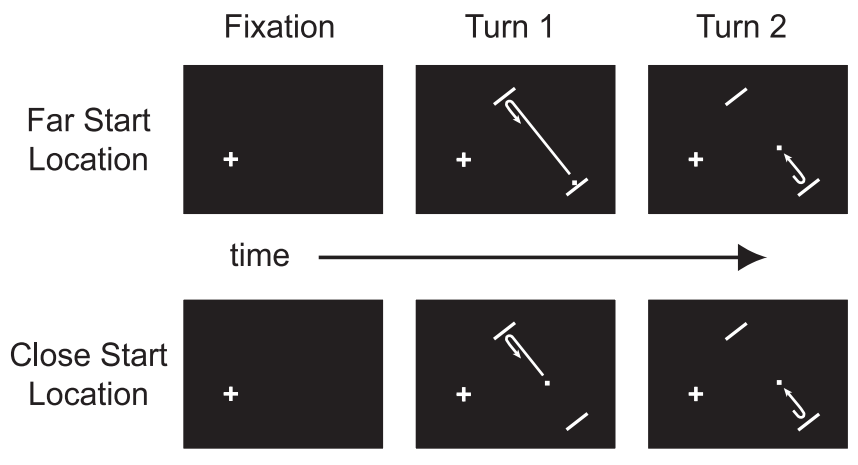

B

ACTIVE

\section{ACTIVE- DELAY}
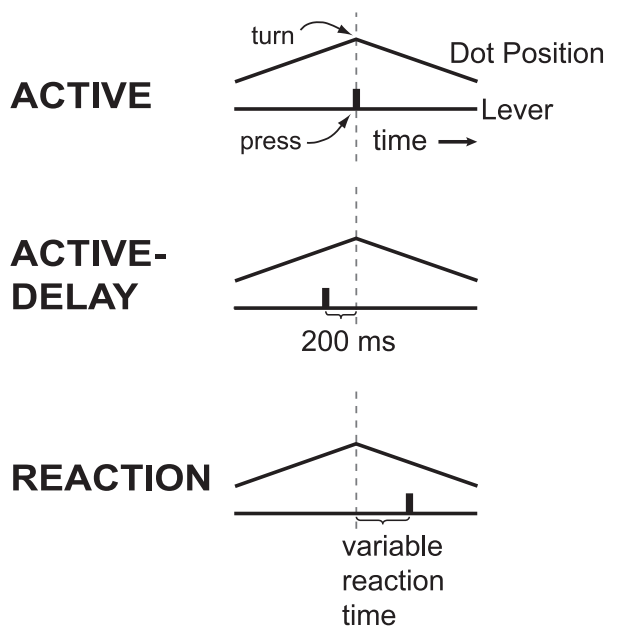

Figure 1. Task. $\boldsymbol{A}$, Schematic of the visual stimulus. The fixation point is shown as a cross. $\boldsymbol{B}$, Three behavioral modes of the main task.

tive for nonreactive movements. These results suggest that the timing of nonreactive movements may be determined by a broadly distributed cortical and subcortical brain network.

\section{Materials and Methods}

Behavioral paradigm. Two male rhesus monkeys (Macaca mulatta; $\sim 10 \mathrm{~kg}$ ) were trained to perform controlled arm movements under visual guidance. The monkeys sat $57 \mathrm{~cm}$ away from a computer monitor $\left(40^{\circ} \times 30^{\circ}\right.$ of visual angle, $75 \mathrm{~Hz}$ refresh, $1152 \times 870$ resolution). Each trial began with the monkeys fixating a small spot on the monitor (Fig. $1 A)$. The animals had to maintain gaze within $0.95^{\circ}$ of this spot at all times or the trial would abort without reward. Five hundred milliseconds after fixation, two parallel bars ("bumpers"; $4^{\circ}$ long, $0.5^{\circ}$ wide, $22^{\circ}$ apart) appeared peripherally. The retinotopic location and orientation of the bumpers were chosen at random for different cells, but were the same on every trial for a given cell. A dot located between the bumpers appeared simultaneously and, after $300 \mathrm{~ms}$, the dot began moving at $13.5 \% \mathrm{~s}$ in a straight path toward one of the bars (the same bar on every trial). The task consisted of three separate blocks of trials: active, active-delay, and reaction (Fig. $1 B$ ).

In the active block, when the monkeys depressed the lever in front of them, the dot reversed its direction of motion immediately (on the next video frame, within $13.3 \mathrm{~ms}$ ). The monkeys' task was to turn the dot each time it approached a bumper during the course of a trial. For a successful trial they were required to turn the dot twice, once near each bumper. The animals were allowed to turn the dot only when it was within $3^{\circ}$ of the bumper for the first turn, and $5^{\circ}$ for the second turn; if the monkeys pressed the lever too early (before the dot entered the requisite zone) or too late (after it hit a bumper), the trial was immediately aborted. If the monkeys responded too late, we continued collecting data for up to 500 $\mathrm{ms}$ after the collision so that the time of the lever press could be accurately recorded. To encourage brisk, consistent movements, we required that the animals raise the lever to its original position within $225 \mathrm{~ms}$ after each depression. If the lever remained down too long, the trial was immediately aborted. The monkeys received a drop of juice as a reward after the second successful reversal (see below). To finish a block, the animals were required to complete 20 correct trials.

The active-delay block was identical to the active block except that the dot turned $200 \mathrm{~ms}$ after the lever press. The monkeys learned to press the lever earlier in these trials to successfully turn the dot. The active-delay block served to dissociate the time of the arm movement from the time of the motion reversal so that neuronal activity could be more clearly related to one or the other event. We refer to the active and active-delay blocks together as the proactive blocks.

In the reaction block, the monkeys no longer had control over the motion reversals. The computer determined when the dot turned and the monkeys were required to hit the lever immediately after noticing a reversal. There were two types of reaction trials, pseudorandomly interleaved. In playback-reaction trials, we replayed trajectories from the active and active-delay blocks. In these trials the animals reacted to the identical motion reversals that they had proactively generated before. In random-reaction trials, we chose the two reversal times at random. These trials were included to make the turn times less predictable, thereby promoting more genuine reactions on all trials. In random-reaction trials, the dot could turn well before the normal turn zone. It could also travel straight through a bumper and turn late. The first random reversal never occurred while the dot was farther than $10^{\circ}$ away from the approaching bumper. Once reaching a $10^{\circ}$ distance, the precise turn time was chosen from an exponential distribution with $\lambda=370 \mathrm{~ms}\left(5.0^{\circ}\right)$. For the second reversal, we waited until the dot traveled $12^{\circ}(889 \mathrm{~ms})$ away from the first turn and then added a different random value drawn from the same exponential distribution. The allowed reaction-time window was $135-500 \mathrm{~ms}$ after a turn. A drop of juice was delivered $400 \mathrm{~ms}$ after the lever was raised on the second press. If at any point the dot exited the screen, the monkeys were rewarded for simply maintaining fixation for an additional $1000 \mathrm{~ms}$; if they pressed the lever in this period, the trial aborted without reward. To finish a reaction block, the monkeys correctly completed 20 playback and 20 random trials, for a total of 40 trials. The 20 playback trials were selected without replacement from the 40 correct trials just completed in the previous active and active-delay blocks.

The blocks were always presented in the following sequence: active, active-delay, reaction. A consistent order was necessary so that we could accumulate active and active-delay trials to replay in the reaction block. We only analyzed neurons for which the animals cycled through all three blocks at least twice, although for 39\% of cells, the animals completed three or four cycles.

To discourage the use of a pure timing strategy in solving the task, we varied the starting location of the dot. On one-half of trials the dot appeared $21.5^{\circ}$ away from the bar, and on the other half of trials, pseudorandomly interleaved, the dot appeared $13.25^{\circ}$ away. Trials in which the dot appeared $21.5^{\circ}$ away are called far-start-location trials, and the others close-start-location trials.

In the active and active-delay blocks, rather than reward the monkeys at a fixed time after the second reversal, a drop of juice was delivered if the dot did not hit either bumper for $3200 \mathrm{~ms}$ after it started to move. This required that the monkeys generate two motion reversals, one near each bumper, but discouraged the tendency of monkeys to press the lever as early as possible in the turn zone. Juice would come no quicker if they turned the dots early.

Whereas one animal (monkey B) performed stereotyped lever presses in all trials, as desired, the other animal (monkey R) tended to roll his arm in a preparatory flourish before lever presses in active and active-delay trials specifically. We could not discourage monkey R from this tendency, and so we retrained him to perform the main task using a touch circuit. His hand rested on a large metal bar. He released and quickly reacquired the metal surface as a surrogate for the lever press. The animal rapidly 
learned the new movement, which completely abolished the premature motions. For most touch-circuit sessions we expanded the reaction-time window by $50 \mathrm{~ms}$, to $135-550 \mathrm{~ms}$, to allow for slightly slower reaction times. All data discussed from monkey R were collected as the animal performed touch releases rather than lever presses.

Electrophysiological technique and data collection. The monkeys were surgically implanted with a head post, scleral search coil, and recording chambers. All surgical procedures followed Harvard Medical School and National Institutes of Health guidelines. The chambers were dorsally positioned at stereotactic coordinates posterior 3, lateral 10 . They were placed in the hemisphere contralateral to the hand used for pressing the lever. Electrophysiological recordings were made from single neurons using tungsten microelectrodes (75 $\mu \mathrm{m}$ shaft diameter; FHC, Bowdoinham, ME) and a guide-tube/grid system. Spike times were recorded with $1 \mathrm{~ms}$ resolution. Horizontal and vertical eye positions were sampled at $200 \mathrm{~Hz}$.

In penetrations targeting the intraparietal sulcus, given our dorsal approach, we often encountered gray matter on the medial bank, then a brief quiet stretch consistent with the sulcus itself, and then gray matter again on the lateral bank. Neurons recorded in the medial bank typically responded to hand-movements and were assigned to area 5. Neurons in the lateral bank were more visually responsive and easily distinguishable from area 5 neurons. Data from lateral bank neurons will be presented separately. Sometimes we encountered gray matter on the gyral surface before we hit the medial bank. Ten of 155 cells assigned to area 5 in this report were more likely in surface cortex, just outside the intraparietal sulcus. (None of the results are substantially altered by excluding these 10 cells.)

Electromyographic (EMG) recordings were made during separate sessions using surface electrodes to measure as broad a signal as possible. The amplified signal was bandpass filtered at $100-5000 \mathrm{~Hz}$ for monkey B and $50-1000 \mathrm{~Hz}$ for monkey R. The EMG signal contained virtually no power past $1000 \mathrm{~Hz}$ so we digitally down-sampled and further low-passfiltered monkey B's data. EMG signals were digitally rectified and averaged for visual presentation and analysis.

Cell categorizations. We classified neurons into two categories based on whether their firing rate increased or decreased at the time of the first arm movement. We compared the spike count in a $500 \mathrm{~ms}$ epoch centered on the time of the first lever-press/touch-release, with a $500 \mathrm{~ms}$ baseline epoch one second earlier (paired $t$ test; $p<0.05$ ). "Burst cells" had significantly more activity in the movement-time window as compared with baseline. "Dip cells" had significantly less activity in the movementtime window as compared with baseline. These classifications were made using correctly executed trials from all three blocks.

Electromyographic latencies and alignment of neuronal responses. For each monkey, we calculated the onset latency of EMG activity relative to the electronically detected lever press or touch release. Examining each rectified, averaged EMG response (see Fig. 5), we calculated the mean and SD of values in a window 900-400 ms before movement. High and low thresholds were set at 3 and 2.3 SDs above the mean, respectively. Starting 400 ms before movement, we analyzed each EMG record onward in time, checking for a significant increase in activity. When a signal crossed the high threshold and remained above the low threshold for at least $12 \mathrm{~ms}$, onset latency was taken to be the instant of the initial highthreshold crossing ( $<1 \mathrm{~ms}$ resolution). For each EMG channel, we generated a single latency measure by averaging the values extracted from the three curves, one from each block. For each monkey, we generated a single EMG latency measure, which was the earliest latency from the four recorded channels.

Because we were interested in neuronal events leading up to the initiation of movement, we often analyzed neuronal responses referenced to EMG onset rather than to touch release or lever press. To do this, one could imagine aligning neuronal data from each trial to the instant at which the muscles began contracting on that trial, but, with a few exceptions, we did not collect EMG data concurrently with neuronal data. As an approximation, we used the averaged EMG data collected in the separate sessions. Specifically, neuronal activity aligned to the lever press or touch release was shifted forward in time by a constant: the EMG latency for that monkey. After this shift, neuronal responses from the two monkeys could be more fairly compared because the two animals had slightly different EMG latencies relative to lever press/touch release (see Results).
To estimate the native variability of EMG onset with reference to the lever press/touch release, we also calculated single-trial EMG latencies. Examining each rectified single-trial EMG response, we calculated the mean and SD of values in a window $900-400 \mathrm{~ms}$ before movement. High and low thresholds were set at 5 and 3 SDs above the mean, respectively. Starting 400 ms before movement, we analyzed each EMG record onward in time, checking for a significant increase in activity. When a signal crossed the high threshold and remained above the low threshold for at least $4 \mathrm{~ms}$, onset latency was taken to be the instant of the initial highthreshold crossing ( $<1 \mathrm{~ms}$ resolution).

Neuronal latencies. We calculated a latency measure for each cell, estimating the time at which neuronal activity first changed relative to the onset of the arm movement. Latency measures referenced to the lever press/touch release were shifted in the manner described above, realigning them to EMG onset, so that data from the two animals could be more properly pooled. We used three different algorithms for extracting neuronal latencies; the first two were adapted directly from the work of DiCarlo and Maunsell (2004). Parameters were chosen so as to yield reasonable latency values as judged by qualitative observation of the data.

In the "maximum-likelihood" algorithm, we followed a method introduced by Commenges and Seal (1985) for estimating latencies in single trials. We aligned spike trains from all correct trials to the time of the arm movement and collapsed the data into a single unified spike train, which contained spike times from all trials. If two or more trials contributed a spike to the identical $1 \mathrm{~ms}$ bin, we ignored additional spikes beyond the first. We then examined spikes in the unified train and found the single spike time that maximized the likelihood that inter-spike intervals before and after that time were chosen from gamma distributions with different means (Commenges and Seal, 1985). In burst cells, we only considered latencies where the mean before the discharge was lower than the mean after, and in dip cells, the opposite. Finally, one needs to specify a time window in which to perform the procedure, preferably a section of data that contains no more and no less than a single abrupt change in firing rate. We found that using an adaptive window tailored to the response of each cell worked best. For each neuron we generated an averagedresponse histogram in the $500 \mathrm{~ms}$ window centered on movement $(20 \mathrm{~ms}$ wide bins), and extracted the time bin with the highest activity (for burst cells) or lowest activity (for dip cells). The latency-analysis window then started $700 \mathrm{~ms}$ before and extended $100 \mathrm{~ms}$ beyond the time of this bin.

In the "least-squares" algorithm, we calculated a rate histogram with 1 $\mathrm{ms}$ bins from all trials. We then tested all time points ( $1 \mathrm{~ms}$ resolution) within the same adaptive window used in the maximum likelihood method. At each time point, we calculated the mean rate before and after that time point, within the adaptive window. We then squared the difference between all $1 \mathrm{~ms}$ bins and their associated mean rate. The time point with the smallest total summed-square error was chosen as the actual latency of the cell. In burst cells, we only queried potential latencies where the mean rate before this latency was lower than the mean rate after, and in dip cells, the opposite.

In the "convolve-threshold" algorithm, spike trains from all correct trials were aligned to the time of the arm movement, convolved with a Gaussian kernel (20 ms SD), and averaged together. We calculated the mean and SD of this averaged-response function in all $1 \mathrm{~ms}$ bins, $1000-$ $500 \mathrm{~ms}$ before movement. High and low thresholds were set at 5 and 3 SDs above the mean, respectively. Starting $500 \mathrm{~ms}$ before movement, we analyzed each averaged response onward in time, checking for a significant change in activity. When a curve crossed the high threshold and remained beyond the low threshold for at least $100 \mathrm{~ms}$, onset latency was considered to be the $1 \mathrm{~ms}$ bin at which the high threshold was initially crossed. In burst cells, we only queried for threshold crossings in the positive direction, and in dip cells, the opposite.

We performed a bootstrap analysis to estimate the SEs of these measures. For each cell, we selected $n$ trials with replacement from the original pool, where $n$ equals the number of trials in the original set. We reran the latency analysis on these trials, generating a new latency value for the cell. We repeated this 100 times, and took the SD of these bootstrapped latencies to be the SE of our original measure (Efron and Tibshirani, 1993; DiCarlo and Maunsell, 2004). 


\section{Results}

\section{Behavior}

We designed our experiment to compare self-timed movements with simple reactive movements. However, it is possible that the animals tried to adopt a uniform behavioral strategy that yielded adequate performance in all three blocks. For example, the animals may not have actually reacted on reaction-block trials, but rather tried to anticipate the reversal of the dot, as on active and active-delay trials. To investigate this possibility, we examined the distributions of arm-movement times in the three blocks (Fig. 2).

Movement-time distributions from active and active-delay trials (first turn only) are shown in Figure $2 A-D$, aligned to the start of dot motion. These distributions were broad, bell-shaped, and centered roughly on the proper time windows for turning the dot (colored regions). Many lever presses/touch releases occurred before or after the correct window, indicating that the animals were not able to use the visual stimulus to perfectly time their movements. The animals did not completely ignore the visual image, however, in that they drew a sharp distinction between the two possible start locations. When the dot started close to the turn zone they moved earlier than when the dot started farther away (Fig. 2, compare $A, B$; compare $C, D)$. Because the two start locations were randomly interleaved from trial to trial, the monkeys must have used visual information, minimally with regard to the initial distance of the dot from the bumper, to grossly guide the timing of their movement.

On a finer time scale, both monkeys appreciated the distinction between active and active-delay blocks. Complete awareness of the difference might have manifested itself as a $200 \mathrm{~ms}$ shift in the average movement times between the two modes. Monkey B exhibited a $122 \mathrm{~ms}$ shift for close-start-locations and a $146 \mathrm{~ms}$ shift for far-start-location trials. Monkey R exhibited 86 and $48 \mathrm{~ms}$ shifts, respectively. Although all differences were highly significant (unpaired $t$ test; $\mathrm{p} \ll 0.01$ ), these undershifts may indicate that the animals tried to split the difference between the two blocks, or failed to fully internalize the extent of the mode difference. This is not critical for our purposes. More important is that the active and active-delay movements distributed broadly with reference to the last sensory trigger available to the animals, the start of dot motion.

Behavioral data from the reaction block are shown in Figure $2 E-L$. On one-half of the reaction trials, we replayed dot trajectories from active and active-delay trials. On the other half, mo-

\section{Monkey B}

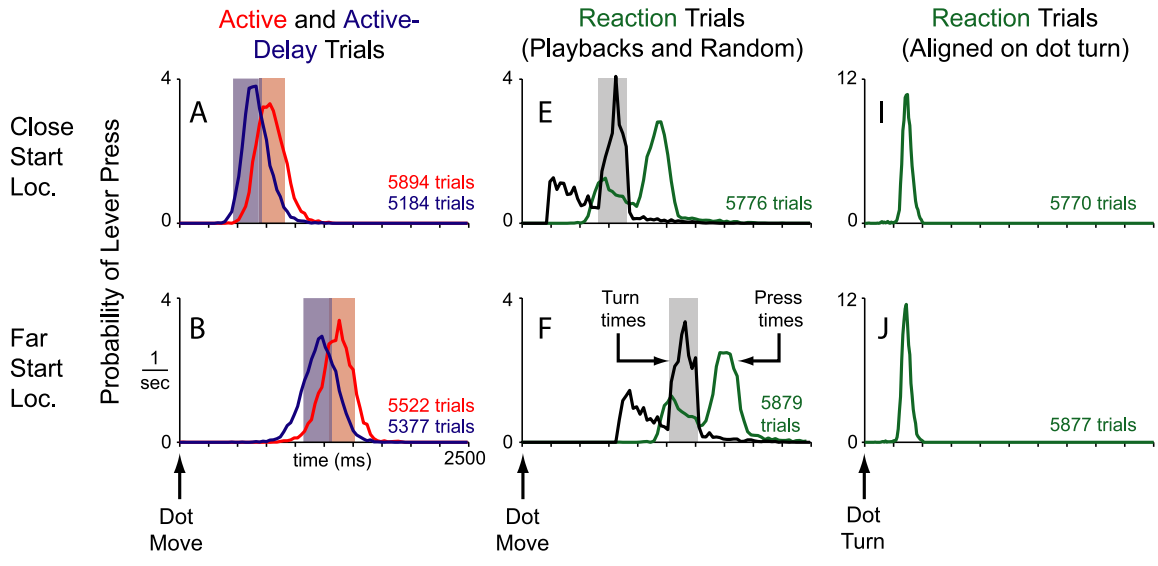

Monkey R

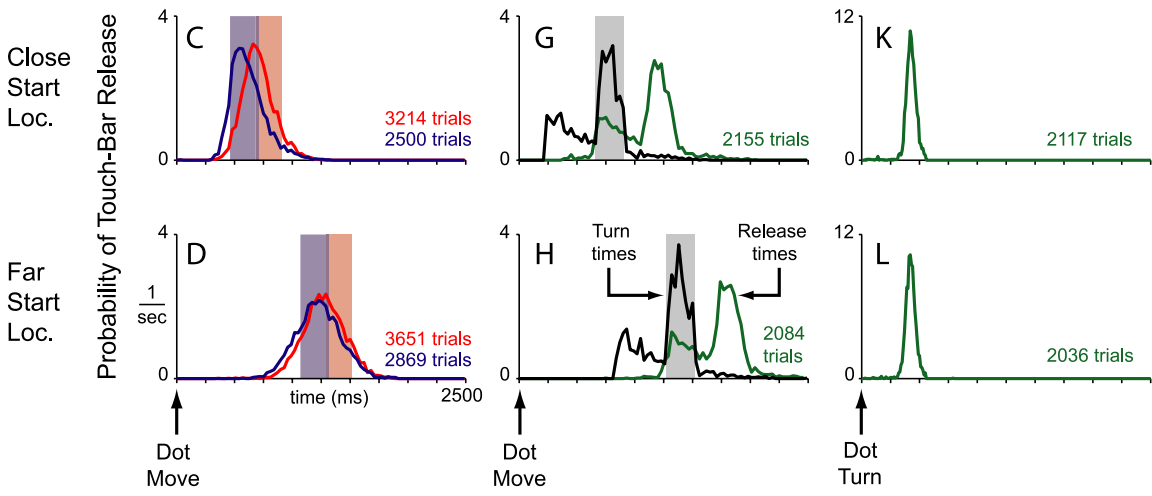

Figure 2. Behavior. $\boldsymbol{A}-\boldsymbol{D}$, Red curves are distributions of arm-movement times in the active block relative to start of dot motion. The light red regions are time windows for correct movements. Because arm movements and dot turns were coincident in the active block, the light red regions also represent time windows in which the dot actually reversed direction. Sections of the red curves that fall outside the light red regions correspond to incorrect movements. The dot never reversed direction on these trials; they ended immediately with no reward. Blue curves are distributions of arm movement times in the active-delay block. Light blue regions are time windows for correct movements. Because dot turns followed arm movements by $200 \mathrm{~ms}$ in this block, motion reversals still occurred in the light red regions. Sections of the blue curves that fall outside the light blue regions correspond to incorrect movements; the dot never reversed direction and the trial ended with no reward. All distributions are from the first turn only and have been normalized to equalize the area under each curve (the $y$ value of each bin was divided by the total number of trials contributing to the distribution, and by the width of the bin, in units of seconds). This normalization converts the curves into density functions, with units of probability/s, which can be properly compared. $\boldsymbol{E}-\boldsymbol{H}$, Distributions of dot-turn times and arm-movement times from the reaction block. The light gray regions indicate the time window for motion reversals in the two active blocks. Because the animals did not sample the turn zone uniformly in correct active and active-delay trials, neither did the playback motion reversals; this is why the black curves take on a jagged form in the gray time windows. Arm-movement time distributions exclude trials that preceded the first correct one in a block. In those trials, the animals inevitably pressed the lever early, not yet realizing that a block change had occurred. Press distributions also exclude late reactions, because the trial ended before an accurate press time could be recorded ( $\sim 1 \%$ of all reactions). Curves in $\boldsymbol{F}$ and $\boldsymbol{H}$ truncate a small number of turns and presses, $<1 \%$. The distributions were normalized as described. $\boldsymbol{I}-\boldsymbol{L}$, Reaction time distributions. The same data as in $\boldsymbol{E}-\boldsymbol{H}$, aligned to dot turn, are shown. There are slightly fewer presses in these distributions as compared with those in $\boldsymbol{E}-\boldsymbol{H}$, because these data do not include the few error presses that preceded a motion reversal. The distributions have been normalized as described, but notice the expanded scale of the $y$-axis. Movement-time variance was much lower in the reaction block as compared with the active and active-delay blocks (i.e., curves in $\boldsymbol{A}-\boldsymbol{D}$ ).

tion reversals occurred at random, exponentially distributed time points in the trajectory (see Materials and Methods). The distributions of turn times and arm-movement times, both referenced to the start of dot motion, are plotted in Figure 2 E-H. The turntime distribution was a straightforward sum of playback turns 
and exponentially distributed turns, as designed. The movementtime distribution shadowed the turn-time distribution, consistent with the animals reacting to the motion reversals in this block. Indeed, by realigning the data to the time of the dot turn (Fig. $2 I-L$ ), it is clear that movements followed turns with a short reaction-time delay.

If the monkeys were anticipating the turns in the reaction block, they might have reacted faster on playback trials, when the dot turned in the requisite proactive-block turn zone $\left(<3^{\circ}\right.$ from the bumper), compared with other trials when the dot turned earlier or later. However, when we examined the monkeys' reaction-times as a function of the location of the dot at the time of the reversal, we found that the animals tended to react with nearly the same latency to all motion reversals. Average reaction times for monkey B were $352 \mathrm{~ms}, 352 \mathrm{~ms}$, and $357 \mathrm{~ms}$ for early, playback, and late dot turns. Average reaction times for monkey $\mathrm{R}$ were $414 \mathrm{~ms}, 407 \mathrm{~ms}$, and $404 \mathrm{~ms}$. These behavioral data suggest that the monkeys were not anticipating the motion reversals in the reaction block.

Furthermore, compared with the proactive-block distributions, movement-time distributions in the reaction block were more compact with reference to the last sensory trigger available to the animals, the dot turn. The SDs of reaction-time distributions were 39 and $42 \mathrm{~ms}$ for close- and far-start-location trials, respectively, from monkey $\mathrm{B}$, and 50 and $53 \mathrm{~ms}$, respectively, for monkey R. In comparison, SDs of movement-time distributions from the proactive blocks were, on average, $129 \mathrm{~ms}$ for monkey B and $166 \mathrm{~ms}$ for monkey R (referenced to start of dot motion). These width differences provide an operational basis for calling movements in the proactive blocks self-timed and movements in the reaction block reactive.

It could be argued, of course, that proactive-block movements were not purely self-timed because the animals likely used some combination of visual analysis and internal timing to solve the task. For example, as the dot approached the bumper it presumably activated neurons with small receptive fields near the bumper. Activation of these neurons could have provided an abrupt cue for movement similar to the dot turns in the reaction block. The crucial point, inferred from the behavioral results, is that whatever the visual signals were in the proactive blocks, they did not provide a cue for movement with as much temporal precision as the motion reversals in reaction trials.

\section{Neuronal activity aligned to dot turn}

We fully characterized 180 neurons in area 5 using the main behavioral task ( 134 cells in monkey B; 46 cells in monkey R). Of this total, 116 burst cells exhibited elevated activity at the time as arm movements as compared with baseline ( 79 cells in monkey B; 37 cells in monkey R), and 39 dip cells exhibited reduced activity with arm movements ( 33 cells in monkey B; 6 cells in monkey R) (see Materials and Methods). Twenty-five neurons had responses that were not significantly modulated and are not discussed further. Burst and dip cells are meant as purely descriptive categories that captured a natural distinction in the data set. Area 5 neurons are known to exhibit direction-selective responses to arm movements (Kalaska et al., 1983; Eskandar and Assad, 2002). It is possible, therefore, that burst and dip cells are simply neurons with opposite preferred directions with reference to the initial direction of arm motion during a lever press or touch release. We will return to this point in the discussion.

Activity from a single burst neuron is shown in Figure $3 A$. Data are aligned to the turn of the dot. Only correct trials are shown. Recall that in active trials the arm movement happened
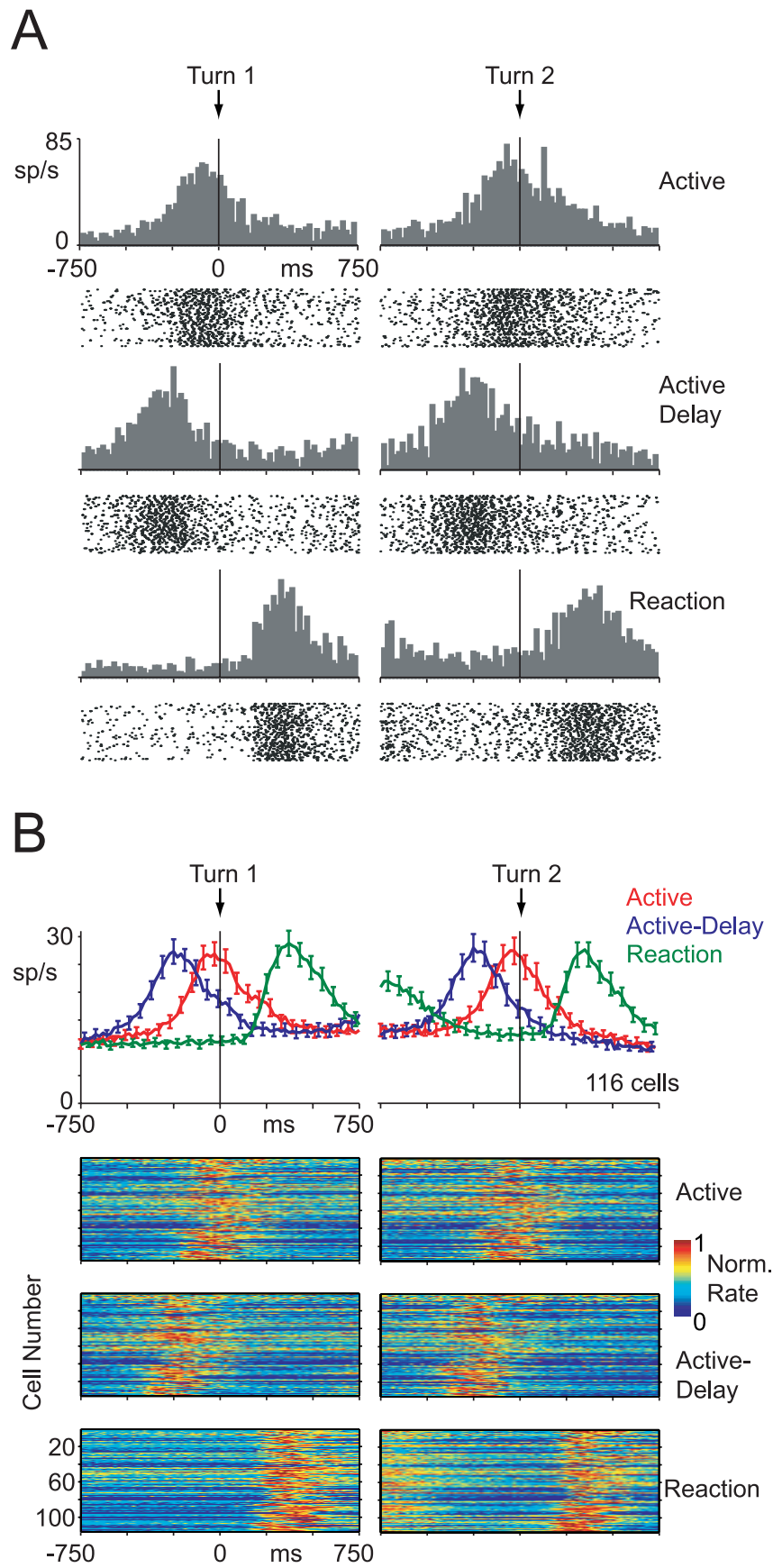

Figure 3. Activity of burst cells, aligned to dot turn. $\boldsymbol{A}$, Single burst cell. Histograms and spike-time rasters from the active block are shown on the first row, the active-delay block on the second row, and the reaction block on the third row. For the reaction block, only data from playback trials are shown. Each tick mark in the rasters corresponds to a single spike time recorded in one correct trial. Histograms use $20 \mathrm{~ms}$ wide bins. $\boldsymbol{B}$, Population activity of all 116 burst cells. Top histograms show population-average activity ( \pm SE). Reaction-block curve includes data from playback- and random-reaction trials. Images below the histograms show data from all the individual cells. Each row in the image corresponds to data from one cell. The bin color corresponds to the average firing rate in that time window, divided by maximum firing rate of the cell in the $1500 \mathrm{~ms}$ surrounding the turn. Normalization was done separately for first-turn and second-turn data. All histograms and images use $20 \mathrm{~ms}$ wide bins.

simultaneous with the turn, in active-delay trials, the arm movement happened before the turn, and in reaction trials, the arm movement happened after the turn. In all three blocks, the burst cell followed this pattern, discharging vigorously whenever the animal moved his arm. Population-averaged responses from all 


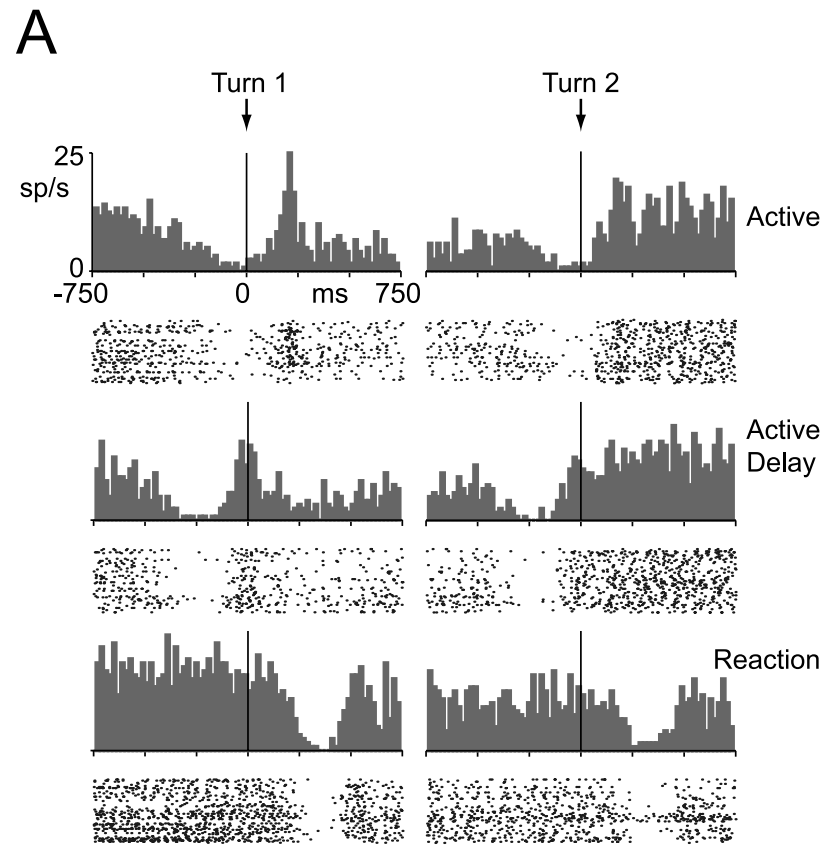

B

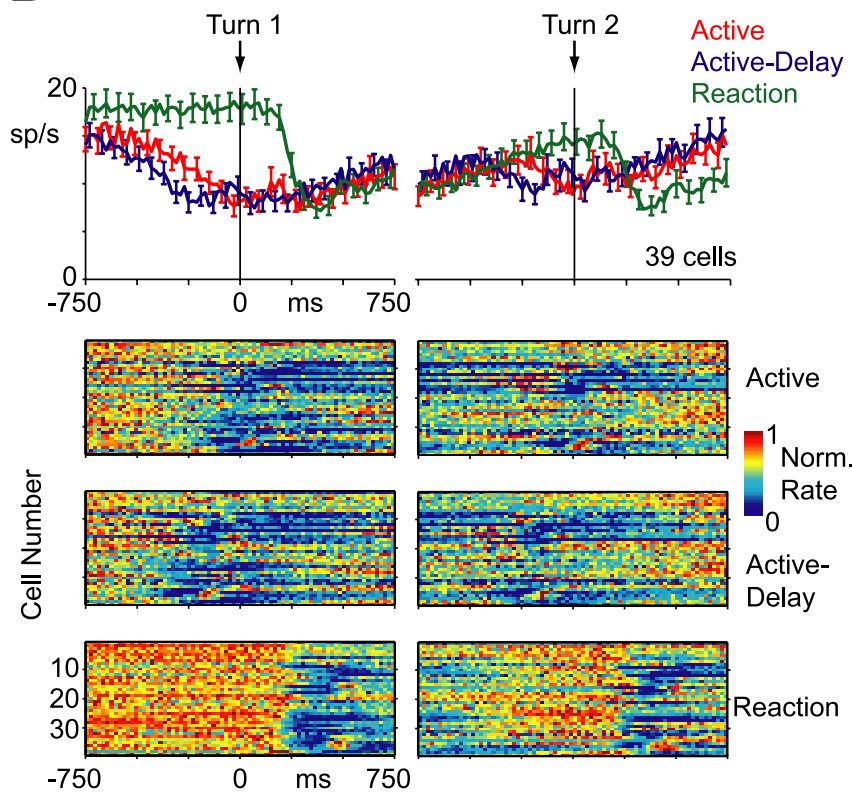

Figure 4. Activity of dip cells, aligned to dot turn. The same layout as Figure 3 is used. Error bars indicate SE.

burst cells are shown in Figure 3B. Area 5 neurons discharged during arm movements with remarkable consistency.

Activity from a single dip neuron is shown in Figure $4 A$. These responses were more complex than those of the burst neuron. In the reaction block, the cell fired tonically until movement, at which point activity dropped off dramatically. The cell then resumed tonic firing at a slightly lower rate until the second movement, at which point activity dropped off again. In the active and active-delay blocks, however, the cell ramped down its firing rate more gradually in the lead up to the first movement. It then never quite recaptured its baseline level before shutting off again around the time of the second movement. After the second arm movement, the neuron resumed tonic firing at a higher level. This cell also exhibited a transient surge in activity after the first dip, perhaps in all three blocks. Many of these basic trends were evident across the population of 39 dip cells (Fig. 4B).

In Figures 3 and 4, we aligned neuronal activity to the time of the dot turn so that it can be readily appreciated that area 5 responses correlated with motor behavior rather than some feature of the visual stimulus.

\section{Electromyographic activity}

To further characterize the dynamics of neuronal activity, the responses should be realigned to the time of the arm movement. However, it was first essential to determine whether the pattern of muscle activations was similar in proactive and reactive trials. Qualitative inspection of the EMG records revealed substantial block differences in muscle tension in the interval between the first and second arm movements. This might explain why neuronal activity in the proactive blocks did not fully return to baseline levels after the first movement, a phenomenon exhibited by both burst cells and, much more dramatically, dip cells (Figs. 3, 4). Because neuronal data preceding the second movement were confounded by these differences in muscle activity, we decided to focus exclusively on the first arm movement in all subsequent analyses. The first movement did not exhibit this confound, as shown below.

Figure 5 shows rectified and averaged EMG activity for the first arm movement. Monkey B made more forceful lever presses during the reaction block as compared with the proactive blocks (green curves vs red/blue curves). Monkey R made virtually indistinguishable touch releases, with perhaps slightly more biceps activity in the proactive modes. However, critically, there were no systematic EMG differences between the blocks in the lead up to the first movement. A region of interest is highlighted in gray. During this interval, the animals' hands rested quiescently on the lever or touch bar in all three blocks.

The earliest EMG signal first exceeded baseline $83 \mathrm{~ms}$ before the lever press for monkey B, and 156 ms before the touch release for monkey R (see Materials and Methods). The discrepancy between the two animals is not surprising because there could be various relationships between muscle dynamics and the time at which the computer detects movement. Certainly the onset of the EMG signal provides a better estimate of the actual onset of movement, in comparison to the time at which a computer registers a lever press or a touch-bar release. We therefore always shift lever-press or touch-release aligned neuronal activity forward in time by $83 \mathrm{~ms}$ for monkey B, and $156 \mathrm{~ms}$ for monkey R. This procedure realigns the data to EMG onset, and allows for a fairer comparison between the two animals.

\section{Burst-cell activity aligned to EMG onset}

Population-averaged neuronal responses from burst cells are shown in Figure 6A, aligned to EMG onset. Starting $\sim 400 \mathrm{~ms}$ before EMG onset, there was a gradual build-up of activity in the active and active-delay blocks, whereas activity in the reaction block remained flat. This difference was highly significant across the population. For each cell, we calculated the mean firing rate during the interval 350-50 ms before EMG onset (Fig. 6A, shaded region). We then calculated a modulation index as $\left(R_{1}-\right.$ $\left.R_{2}\right) /\left(R_{1}+R_{2}\right)$, where $R_{1}$ is the mean rate on active and activedelay trials combined, and $R_{2}$ is the mean rate of reaction trials. The index ranges from -1 to 1 , with positive values indicating higher activity in the proactive blocks and negative values indicating higher activity in the reaction block. The distribution of 116 indices was shifted significantly positive from zero $(t$ test; $p<$ 
EMG Activity
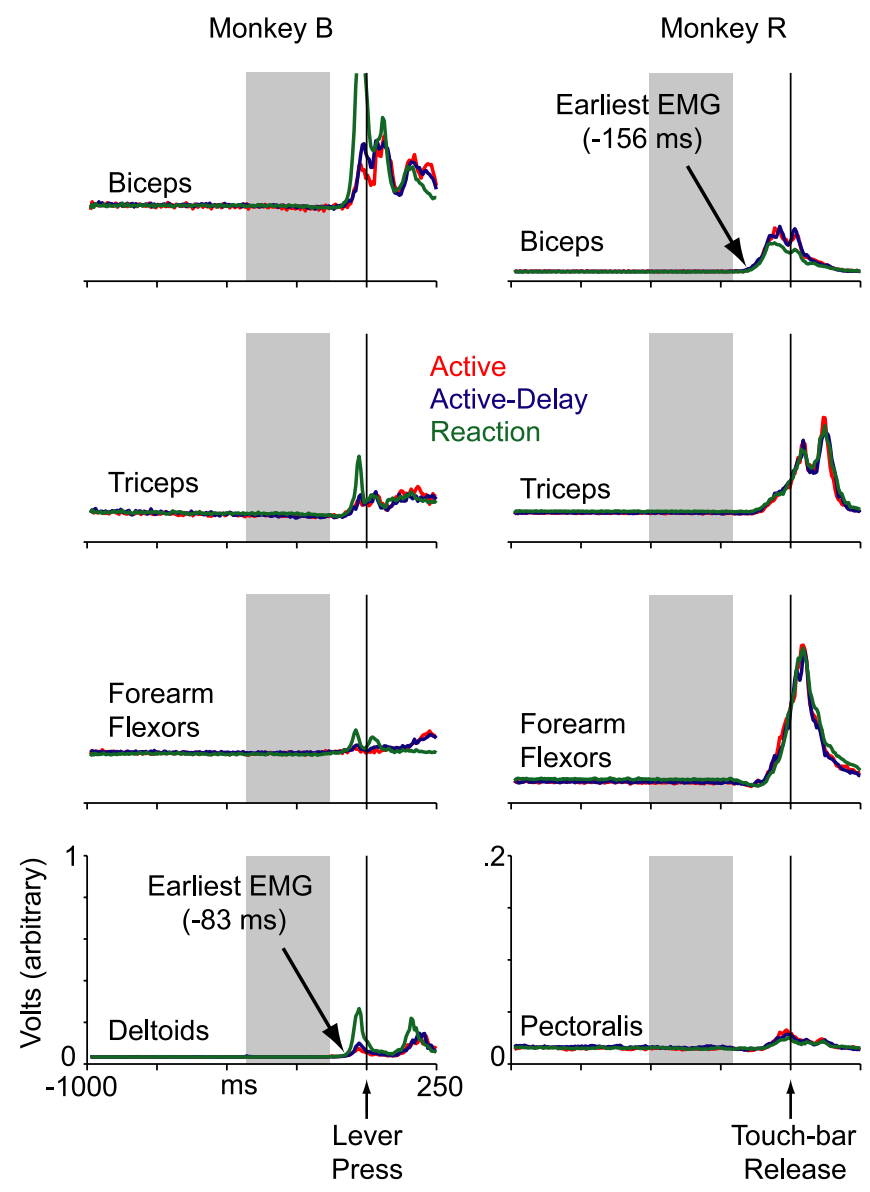

Figure 5. Electromyographic activity. In monkey $B$, data were collected from the biceps, triceps, forearm flexors, and deltoids. In monkey R, data were collected from the biceps, triceps, forearm flexors, and pectoralis muscles. Reaction-block curves include data from playback- and random-reaction trials.

0.01) (Fig. 6A, inset). Analyzing the neurons individually, 35 had significantly more activity in the proactive blocks and only 10 had significantly less $(t$ test; $p<0.01)$. Furthermore, the difference between proactive and reactive blocks was only evident immediately before EMG onset. We calculated the identical modulation index in five nonoverlapping, 300-ms-wide bins spanning 1250 $\mathrm{ms}$ before movement to $250 \mathrm{~ms}$ after movement (Fig. 6B). Mean modulation indices were not significantly different from zero except in the one bin mentioned above, immediately preceding EMG onset (Fig. $6 C$ ) $(t$ test; $p<0.01)$. Note that the shaded time window in Figure $6 \mathrm{~A}$ is identical to the highlighted time epochs in the EMG data (Fig. 5). Proactive arm movements were associated with increased firing rates in the lead-up to movement, well before any detectable changes in muscle activity.

The rightmost histogram of Figure $6 B$ captures the perimovement activity of the neurons (i.e., during a $300 \mathrm{~ms}$ window starting $50 \mathrm{~ms}$ before EMG onset). Although the mean modulation index in this epoch did not differ significantly from zero, more individual cells exhibited significant differences in activity between the proactive and reactive blocks than would be expected from chance. Because the neurons were firing at a higher rate in this time epoch, it is not surprising we could detect statistically significant differences more readily than in the earlier time windows (Lee and Assad, 2003). These modulations could reflect

\section{Burst Cells}
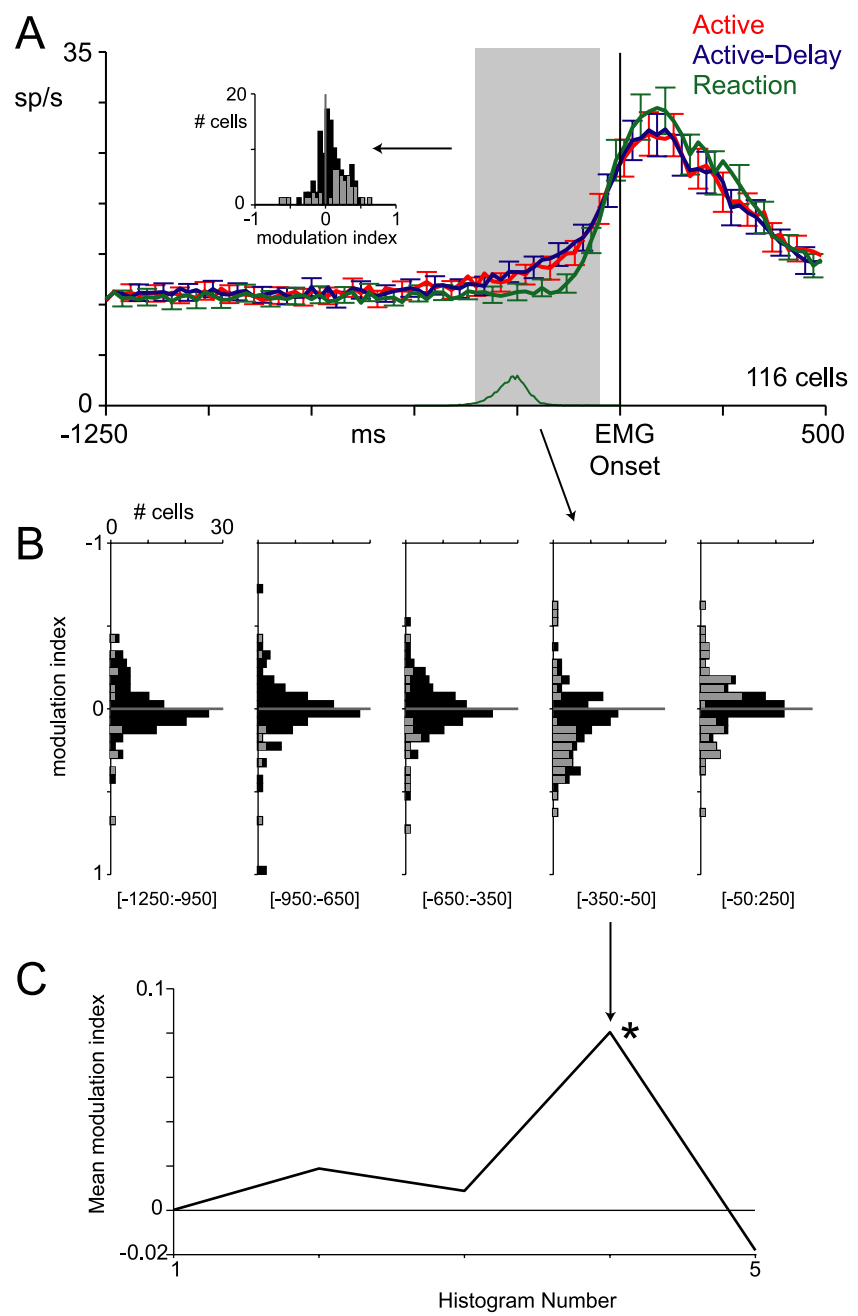

Figure 6. Population activity of burst cells, aligned to EMG onset. $A$, Population-averaged histogram ( $\pm \mathrm{SE}$ ) of 116 burst cells. Reaction-block curve includes data from playback- and random-reaction trials. The distribution of dot-turn times in the reaction block is shown in the small green curve preceding EMG onset. The gray region highlights a $300 \mathrm{~ms}$ wide time window, which starts 350 ms before EMG onset. For each cell, we calculated the index: $\left(R_{1}-R_{2}\right) /\left(R_{1}+R_{2}\right)$ in this window. $R_{1}$ denotes the mean rate of active and active-delay trials combined. $R_{2}$ denotes the mean rate of reaction trials (including random reactions). The inset shows the distribution of modulation indices. Light-gray bins indicate cells with individually significant spike-rate differences between the blocks (unpaired $t$ test; $p<0.01$ ). $\boldsymbol{B}$, Distribution of modulation indices comparing active and active-delay trials with reaction trials. For each cell, we calculated the same index as in $A$, at various $300 \mathrm{~ms}$ wide time windows, as indicated. $C$, Mean modulation index over time. The mean modulation index of each histogram in $\boldsymbol{B}$ is plotted. The asteriskindicates a mean value significantly different from zero.

some specificity in area 5 neurons, with some cells more involved in reactive movements and others more involved in self-timed movements. Alternatively, they could also be related to differences in muscle activity. Indeed, slightly more of the significant indices were negative than positive ( 36 vs 24 ), consistent with the general trend toward more perimovement EMG activity in the reaction block for monkey $B$. The perimovement indices were only weakly correlated with the indices observed before movement initiation $(r=0.27$, comparing values in the fourth and fifth histograms of Fig. 6B); thus, modulations observed during the execution of movements were not strongly related to modulations observed during the initiation of movement, in which we are more interested. 
Nevertheless, the general pattern of elevated activity in the lead-up to EMG onset and lowered activity (albeit nonsignificantly) during the actual movement suggests that the responses on proactive trials may have been a smeared version of responses on reaction trials. One possibility is that the onset of EMG activity, in reference to the lever press/touch release, was more variable in the active and active-delay blocks as compared with the reaction block. This would tend to smear the averaged data in a manner that elevates activity early and reduces activity late, much like what happens to a step function when convolved with a Gaussian. The averaged EMG data from Figure 5 argue against this interpretation because EMG onsets seem similar in proactive and reactive conditions. Nonetheless, we extracted trial-by-trial estimates of onset latencies for EMG activity (see Materials and Methods), and examined the variability of these distributions in the three blocks. Data from monkey R's biceps, triceps, and forearm flexors had high enough signal-to-noise ratios to allow for a reliable trial-by-trial latency analysis. We found that the means and SDs of the EMG-onset distributions were very similar across the three blocks (active mean $\pm \mathrm{SD}$, active-delay mean $\pm \mathrm{SD}$, reaction mean $\pm \mathrm{SD}$; all values are in $\mathrm{ms}$ ): biceps, $-76 \pm 28$, $-77 \pm 35,-76 \pm 27$; triceps, $10 \pm 61,11 \pm 76,10 \pm 64$; forearm flexors, $-1 \pm 44,-7 \pm 50,5 \pm 43$, respectively. Our results are thus unlikely to have resulted from block differences in the variability between EMG onset and the time at which the computer detects movement.

Another possibility, however, is that rather than reflecting external jitter between EMG onset and lever press/touch release, our proactive data might reflect internal jitter between neuronal onset and EMG onset. That is, cells might have fired the same movement-related burst on all trials, but in proactive blocks, the bursts may have been inconsistently aligned, trial-to-trial, with reference to EMG onset. This would also tend to smear the averaged data in a manner that elevates activity early and reduces activity late. To examine this possibility, we took the average reaction-block curve of Figure $6 \mathrm{~A}$, shifted it backward and forward randomly 1000 times, and averaged the resulting set of misaligned curves. We chose shifts from a normal distribution with a mean of 0 and an SD of $160 \mathrm{~ms}$. With these parameters, the misaligned and averaged reaction-block curve was able to capture the premovement rise of the proactive data, but it greatly overestimated the decrement in perimovement activity (Fig. 7). Burstcell responses on proactive trials, therefore, were not merely misaligned reactive responses. Instead, proactive responses are better conceptualized as largely similar to reactive responses, except for an additional firing-rate elevation specific to the premovement time window. These trends were even more evident in dip cells.

\section{Dip-cell activity aligned to EMG onset}

Population-averaged responses from dip cells are shown in Figure $8 \mathrm{~A}$. In the reaction block, activity remained at baseline until $\sim 150$ ms before EMG onset, at which point there was an abrupt drop-off in firing. In contrast, on proactive trials, there was a gradual reduction in activity starting $\sim 400 \mathrm{~ms}$ before EMG onset. This premovement dynamic mirrored the modulation observed in the burst cells. Perimovement activity, however, was quite similar in all three blocks. Tonic activity at the start of the trials, even before fixation, was also slightly lower on proactive trials.

These trends were consistent across the population of $39 \mathrm{dip}$ cells. We calculated modulation indices as in Figure 6 (Fig. $8 \mathrm{~A}$, inset, $B, C)$. Unlike for burst cells, the mean index for dip cells was significantly different from zero as early as $1250 \mathrm{~ms}$ before EMG onset $(t$ test; $p<0.01$ ). In the time window immediately preced-

\section{Misalignment Model}
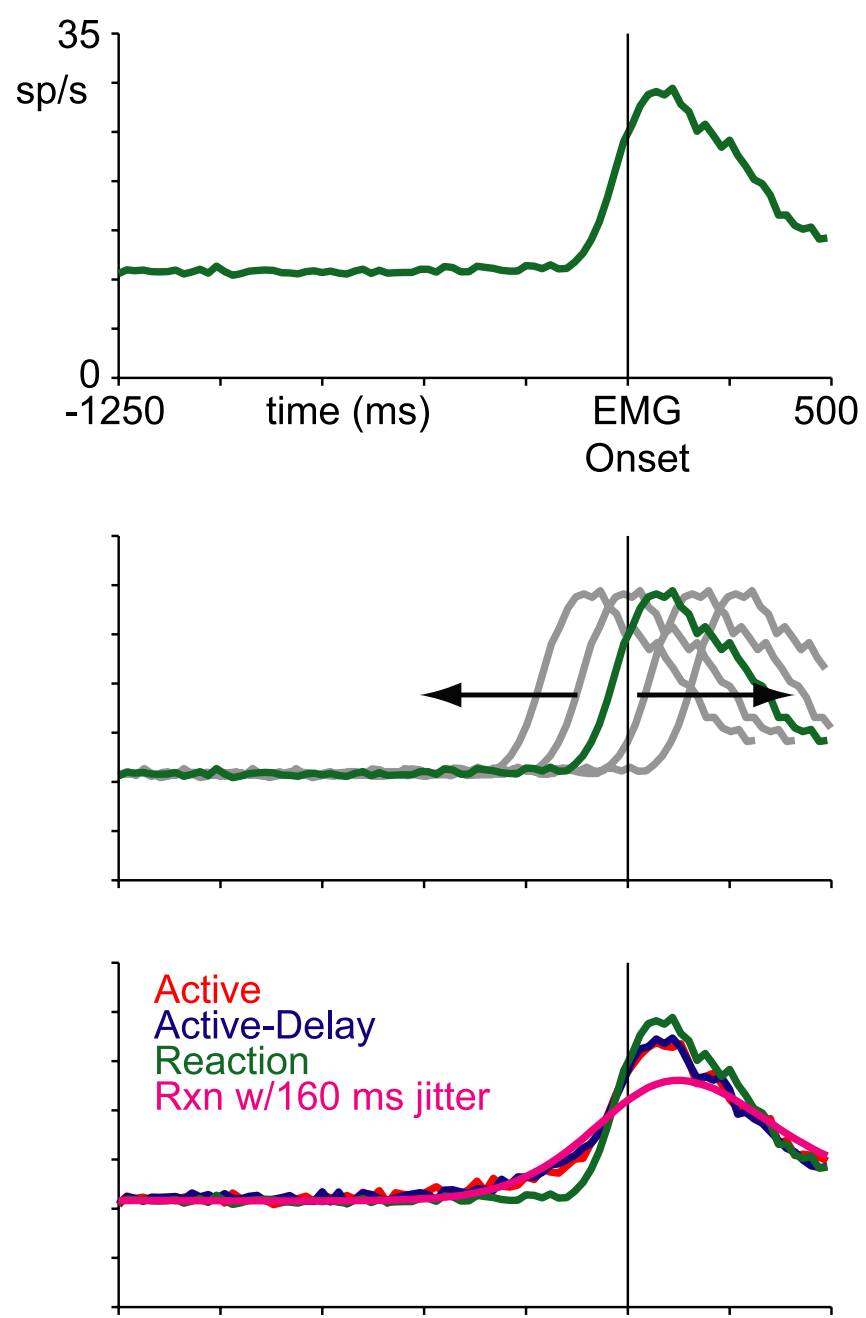

Figure 7. Misalignment model. Top, Reaction-block data from Figure 6. Middle, Schematic of back-and-forth shifting. Bottom, Same data as in Figure 6, with a magenta line showing the shifted and averaged reaction-block curve.

ing EMG onset (shaded region), the difference was especially pronounced; 23 cells had significantly less activity in the proactive blocks than the reaction block and only two had significantly more (unpaired $t$ test; $p<0.01$ ), indicating that an additional or stronger modulation occurred in this time window. Perimovement indices were not significantly different from zero ( $t$ test; $p=0.72 ; 300 \mathrm{~ms}$ window starting $50 \mathrm{~ms}$ before EMG onset).

\section{Two monkeys}

The premovement differences in activity between proactive and reaction blocks were present in both monkeys. Figure $9 A$ shows population-averaged responses from the two animals separately. The effects were not as clear in monkey $\mathrm{R}$, perhaps because of the smaller sample size. Recall that monkey B made more forceful presses in the reaction block, and monkey R made slightly more forceful presses in proactive blocks (Fig. 5, EMG data). However, for both animals, the average premovement neuronal activity was larger for proactive than reaction trials. This argues that the premovement modulations were not related to differences in the upcoming force of movement, but rather reflect something about how movements are initiated in the two types of trials. In addition, 


\section{Dip Cells}

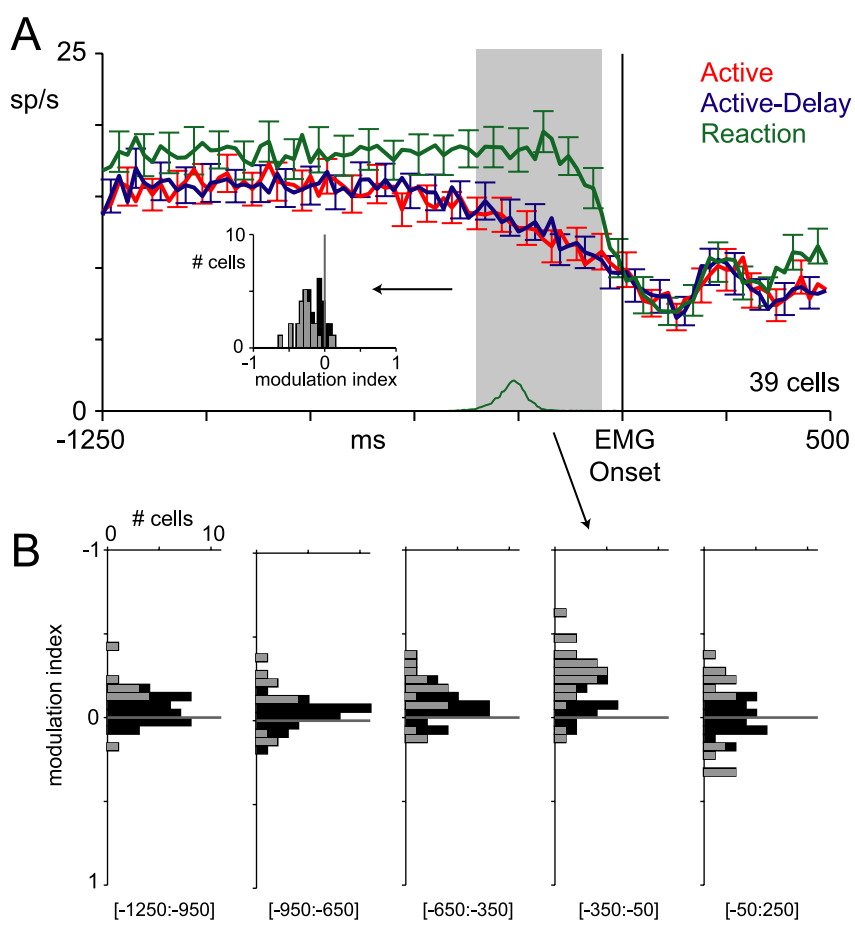

C

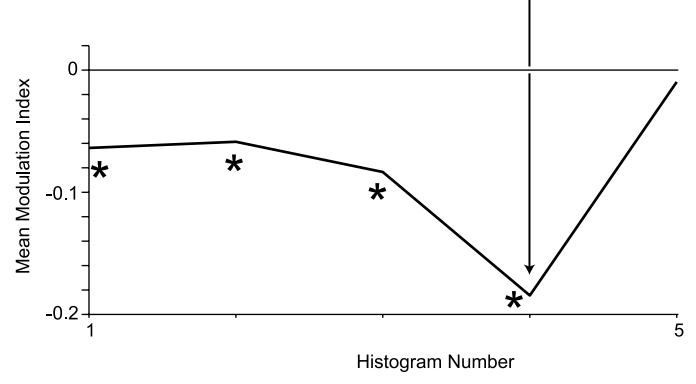

Figure 8. Population activity of dip cells, aligned to EMG onset. The same layout as Figure 6 is used. Error bars indicate SE. The asterisks indicate a mean value significantly different from zero.

the tonic component of activity seems absent in the dip-cell data from monkey R. This could be attributable to the small sample size, or slight differences in behavioral strategy between the monkeys.

\section{Other possible explanations for the premovement changes in} neuronal activity

Thus far, we have lumped together reaction-block data from both playback- and random-reaction trials. However, only on playback-reaction trials did the dot turn at the same visual locations as on active and active-delay trials; on random-reaction trials, for example, the dot often turned earlier. If area 5 activity ramped gradually as the dot approached the turn zone in all blocks, the reaction-block curves could have appeared flat artifactually, because we included early turns in our average. To examine this issue, we plotted reaction-block responses split by the location of the dot at the time of the motion reversal. Neuronal activity was remarkably similar for movements occurring in reaction to early turns, playback turns, and late turns (Fig. 9B). This is consistent with the similar reaction times we found for these three categories of trials, mentioned earlier. Moreover, we repeated the analyses of Figures 6 and 8, comparing only the
A
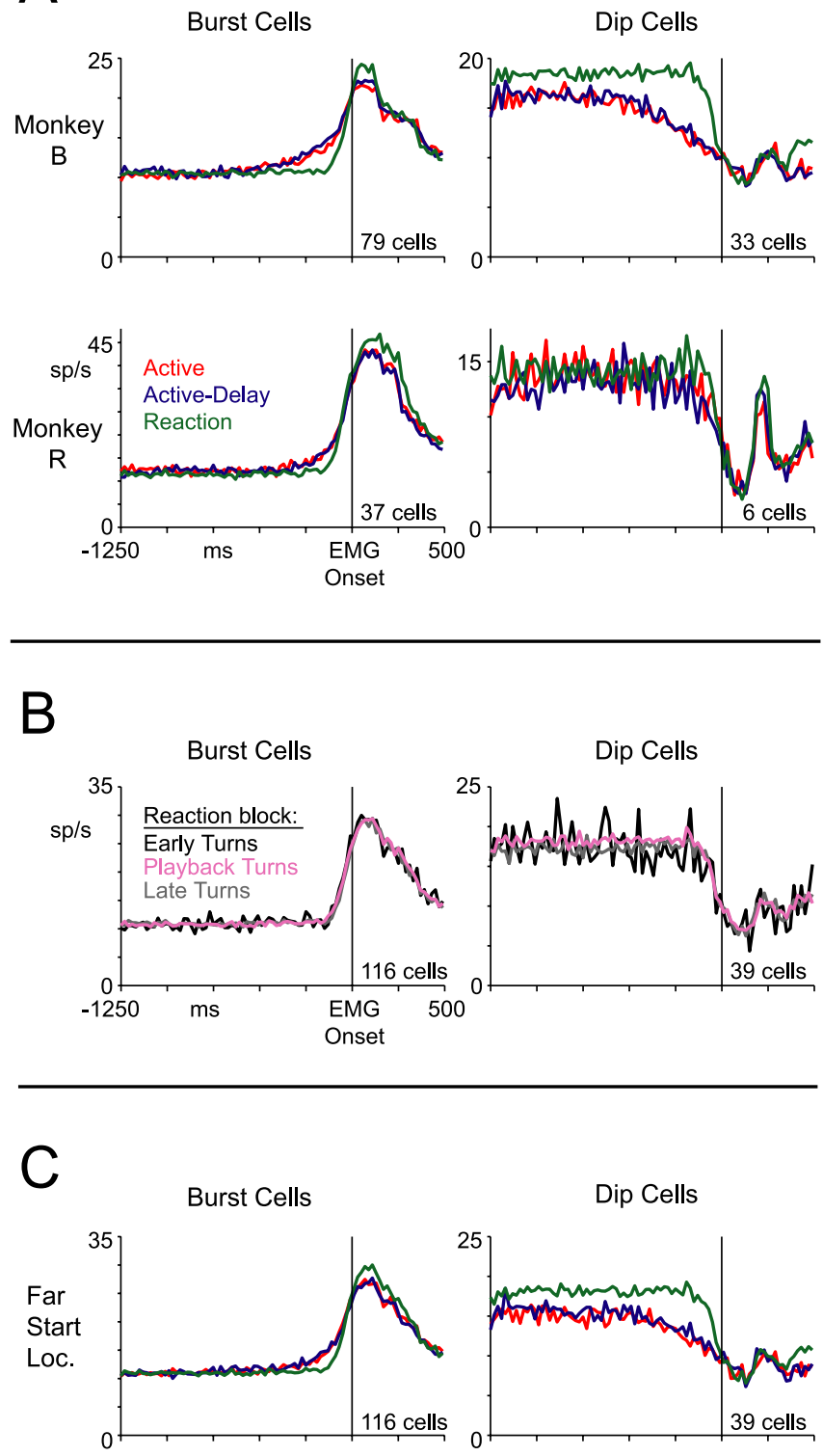

Dip Cells

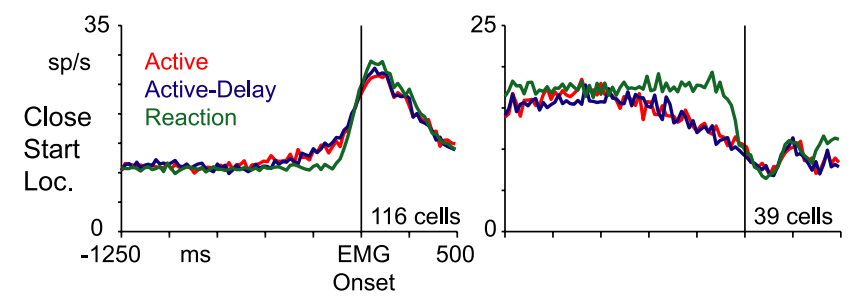

Figure 9. Controls. $\boldsymbol{A}$, Population-averaged histograms for each monkey shown separately. $\boldsymbol{B}$, Population-averaged histograms in the reaction block split by turn location. Early turns occurred before the active-block turn zone. Playback turns occurred within the active-block turn zone. Late turns occurred after the active-block turn zone. C, Population-averaged histograms for each start location shown separately.

playback-reaction trials with the proactive data, and obtained very similar results (data not shown).

Another possibility is that the gradual change in activity in the proactive blocks represents the elapsed time from the start of dot motion. If so, the activity should look different between far- and close-start location trials, because an additional $611 \mathrm{~ms}$ elapsed before the dot reached the requisite turn zone on far-startlocation trials. Figure $9 C$ shows population-averaged responses 

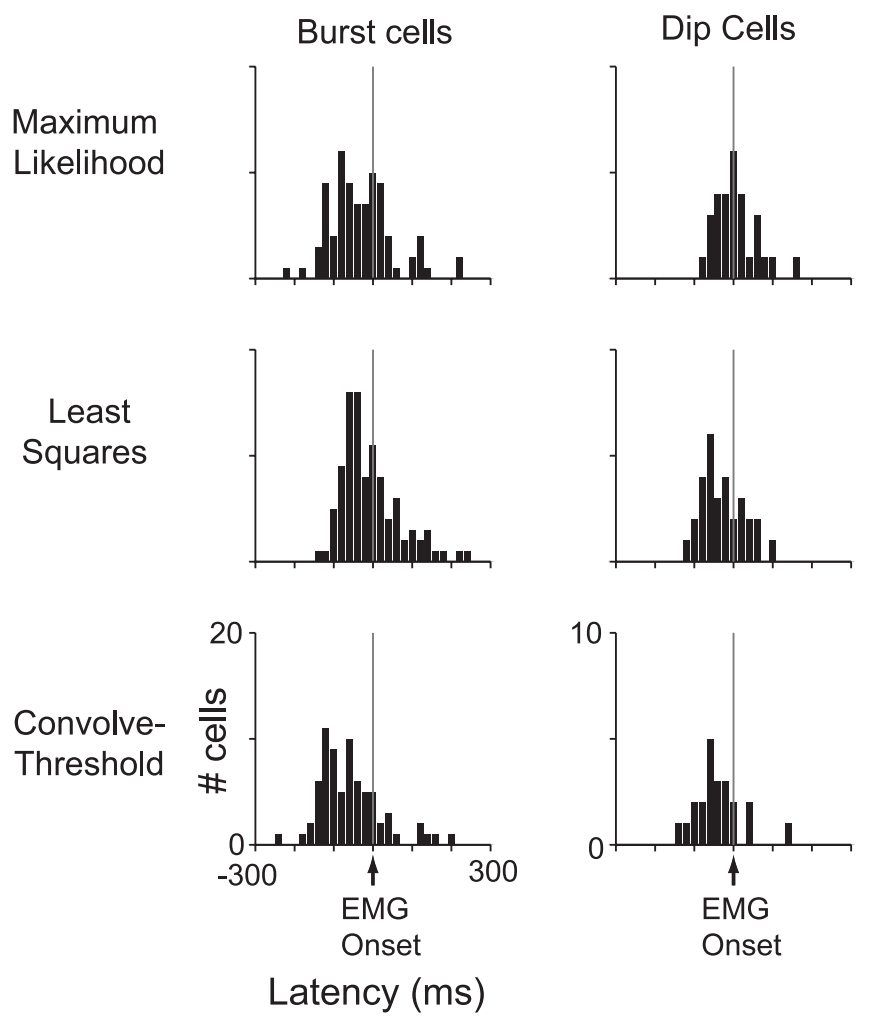

Figure 10. Latencies. Distributions of neuronal response latencies using three different algorithms are shown. Only cells with well estimated latencies are displayed (SE $<40 \mathrm{~ms}$ ). 0 f 116 burst cells, latencies are shown from 86 neurons using maximum-likelihood, 99 neurons using least-squares, and 80 neurons using convolve-threshold. 0 f 39 dip cells, latencies are shown from 29 neurons using maximum-likelihood, 30 neurons using least-squares, and 22 neurons using convolve-threshold.

split by start location. Gradual premovement changes in activity were evident for both trial types. In close-start-location trials, the premovement activity in burst cells was perhaps slightly enhanced, whereas the tonic component in dip cells seemed weaker $750 \mathrm{~ms}$ before the arm movement. Notwithstanding these subtle differences, the gradual changes in activity in the proactive blocks did not appear to signal elapsed time because the changes were clearly linked to the time of the arm movement and not to the start of the motion of the dot.

\section{Neuronal Latencies}

For area 5 to play a role in movement initiation, at minimum, single neurons need to activate before movements are initiated. It is apparent from Figures $6 \mathrm{~A}$ and $8 \mathrm{~A}$ that our population of area 5 cells, on average, activated $\sim 150 \mathrm{~ms}$ before the earliest detected EMG signal. To determine when single neurons activated in reference to movement, we extracted a neuronal latency for each cell, with reference to EMG onset. We only used reaction trials in estimating neuronal latency because cells exhibited the most abrupt firing rate changes in these trials. Moreover, reaction trials should yield the latest and, thus, most conservative latency estimate for each cell. We determined neuronal latency using three different algorithms to measure when activity first changed from baseline: maximum-likelihood, least-squares, and convolvethreshold (see Materials and Methods). Figure 10 shows latency distributions from all neurons that furnished reliable $(\mathrm{SE}<40$ $\mathrm{ms}$ ) estimates. With maximum-likelihood, $74 \%$ of burst cells and $74 \%$ of dip cells were included. With least-squares, $85 \%$ of burst cells and $77 \%$ of dip cells were included. With convolve- threshold, $69 \%$ of burst cells and $56 \%$ of dip cells were included. Independent of the algorithm used, it is clear that many, if not most, burst cells and dip cells became active before the earliest detected EMG signal, consistent with these cells playing a role in movement initiation.

\section{Discussion}

Our goal was to better characterize the neuronal processes of self-timed versus reactive movements. We defined self-timed movements in purely operational terms: those drawn from a movement-time distribution that is significantly wider than a standard reaction-time distribution. The monkeys' presses/releases in the proactive blocks met this restricted definition, although the animals might have used visuospatial information as well. We found that area 5 neurons in parietal cortex, although generally active during all task-relevant movements, responded differently before self-timed versus reactive movements. Burst neurons ramped up their firing rates hundreds of milliseconds before self-timed actions. Dip neurons showed the opposite trend. In contrast, the same cells displayed flat response profiles in the lead up to reactive movements.

The gradual changes in firing were not related to differences in the visual input. Active and active-delay responses overlapped perfectly when aligned to EMG onset, although the visual stimulus differed between the blocks under this alignment. The modulations were also not related to detectable differences in muscle activity. Neither animal exhibited EMG signals above baseline as the neurons gradually increased or decreased their firing. An apparent concern is that monkey $\mathrm{B}$ performed demonstrably more forceful lever presses in the reactive block as compared with the proactive blocks. However, for two reasons, it is unlikely that the neuronal effects reflect a premotor signal related to the force of an upcoming movement. First, monkey R exhibited the same neuronal trends, and made, if anything, more forceful presses in the proactive blocks (Figs. 5, 9A). Second, one might expect changes in neuronal activity predictive of force to be more pronounced in the lead-up to more forceful movements. We observed the opposite trend in monkey B, where premovement increases in activity were stronger on proactive trials, in which the animals made less forceful movements. We conclude that the gradual changes in premovement activity, which are unrelated to measurable sensory or motor variables, instead reflect an internal mechanism for the initiation of self-timed actions.

\section{Positive feedback loops and initiation of action}

Interestingly, the modulations observed in area 5 bore a striking resemblance to modulations observed in the putamen by Lee and Assad (2003). In Figure 11, we show the data side by side. Burst cells in parietal cortex and phasically active neurons in the putamen both exhibited large perimovement responses preceded by smaller ramp-like modulations evident in self-timed trials specifically. A hypothesis considered by Lee and Assad, consistent with the data presented here, is that initiation of concerted movements might be driven in part by positive-feedback connections between the cortex and basal ganglia. This would imply a systemslevel threshold for initiating action. Self-timed movements might be driven by network activity that reaches threshold gradually, whereas an abrupt sensory trigger might drive the system to threshold more quickly. Our results confirm that neocortical neurons could contribute to self-initiated movements and surprisingly point to the parietal cortex, a region more commonly associated with visually cued or visually guided movements rather than self-initiated movements. 

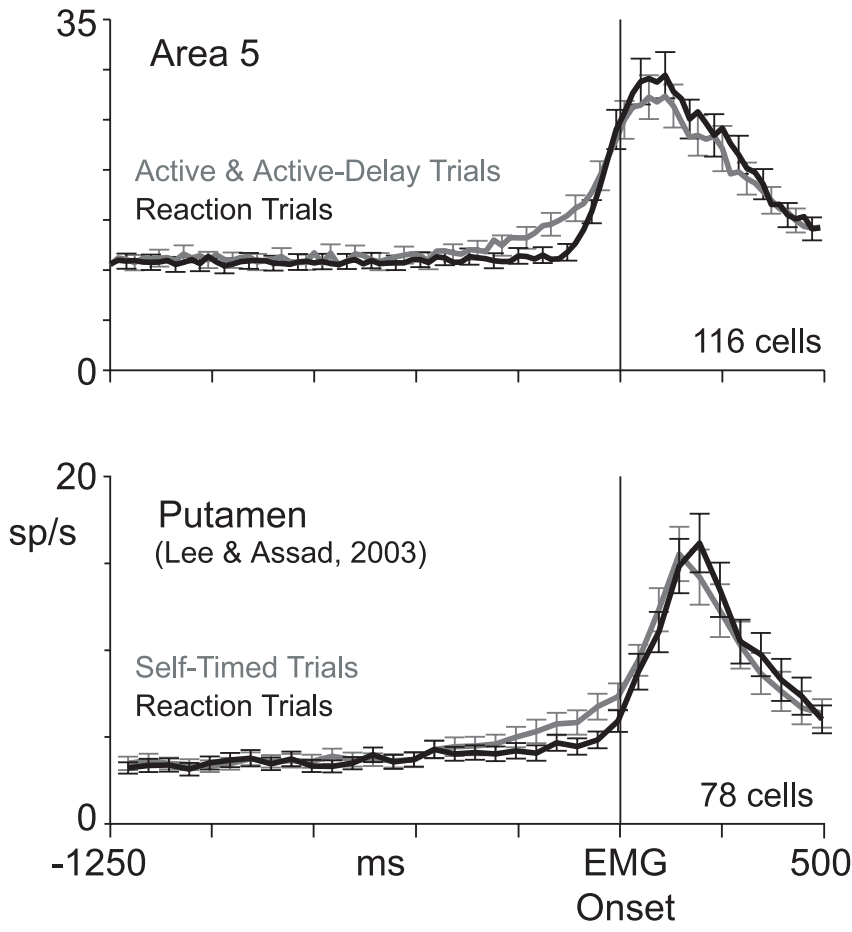

Figure 11. Area 5 versus putamen. Top, Population-averaged histograms from the current data set. Active and active-delay trials were pooled together and averaged. Bottom, Population-averaged data from phasically active neurons in the putamen (Lee and Assad, 2003). Original data have been realigned to earliest EMG onset, as determined by eye using the EMG curves of Figure 4 in Lee and Assad (2003). Error bars indicate SE.

In support of the positive-feedback idea, it is becoming increasingly clear that the cerebral cortex forms a series of closed anatomical loops with the basal ganglia and cerebellum (Alexander et al., 1986; Hoover and Strick, 1999; Kelly and Strick, 2003). Middleton and Strick (2000) have pointed out the need to define the computations this sort of anatomical substrate might support. One simple process that a closed-loop architecture might be good for is the amplification of responses through positive feedback (Sanger, 2003). If the set of synapses in the loop are net excitatory, the network could produce all-or-nothing firing behavior in neurons throughout the system. All-or-nothing responses might be important for generation of concerted movements, where muscles need to act in unison at the moment of initiation. It is not known whether area 5 makes closed-loop connections with the basal ganglia or cerebellum, but strong projections to the putamen, premotor cortical areas, and even the spinal cord have been discovered (Yeterian and Pandya, 1993; Johnson et al., 1996; Matelli et al., 1998; Marconi et al., 2001). Area 5 neurons could thereby participate in a positive feedback process, perhaps evident throughout the entire motor system (Prut and Fetz, 1999). Consistent with a role in movement initiation, we found that many area 5 neurons responded $100 \mathrm{~ms}$ or more before detectable EMG activity, even in the reaction block (Fig. 10). Early responses of this sort have been reliably observed in area 5 (Seal et al., 1982; Kalaska et al., 1983; Burbaud et al., 1991).

Burst cells and dip cells: descriptive names or functional categories?

Burst cells exhibited gradual premovement increases in activity on proactive trials as compared with reaction trials, whereas dip cells exhibited the opposite trend. Because we did not categorize neurons as burst or dip based on these premovement differences, the opposite sign of premovement modulations in burst and dip cells suggests that the two groups are functionally distinct. To the extent that our premovement modulations are similar to delayperiod modulations in delayed-response tasks, this result might be expected. For example, if one assumes that burst cells and dip cells are simply neurons for which the initial axis of movement corresponded to their preferred directions and null directions respectively, it is known that delay-period modulations in area 5 generally have the same sign as the perimovement response (Crammond and Kalaska, 1989).

However, there was a curious difference between our two classes of cells. Burst cells exhibited firing-rate elevations related to self-timed movements, which started $\sim 400$ ms before EMG onset (Fig. 6). Dip cells mirrored this ramping effect but, additionally, they exhibited a baseline modulation starting far before, as early in a trial as we could measure (Fig. 8). This asymmetry is not expected if both groups of cells serve the same function. In the oculomotor system, many neurons in the brainstem, superior colliculus, and cerebral cortex respond tonically during fixation and shut off briefly during eye movements (Munoz and Wurtz, 1993; Hanes et al., 1998; Scudder et al., 2002). It has been proposed that activity in these neurons might be critical for maintaining gaze. By analogy, at least some of our dip cells, rather than simply encoding the null-direction movement, might instead be "hold neurons" for the skeletomotor system. In the reaction block, a stronger hold signal might be needed because the animals are required to actively prevent self-timed movements while waiting for a motion reversal.

Self-timed ramping activity versus delay-period modulations. Previous work examining cortical activity for self-initiated movements has emphasized the long lead time between neuronal responses and the start of action. Certain cells in premotor cortex, for example, exhibit firing-rate elevations $1 \mathrm{~s}$ or more before movement (Okano and Tanji, 1987; Romo and Schultz, 1992). This time course is broadly consistent with that of the readiness scalp potential recorded in human and monkey subjects as they prepare internally generated actions (Gemba and Sasaki, 1984; Deecke, 1996; Sirigu et al., 2004). In area 5 cells, however, we observed ramping activity that began $\sim 400 \mathrm{~ms}$, not $1000-2000$ $\mathrm{ms}$, before EMG onset (in dip cells we observed a baseline shift as well, which we consider to be functionally separate).

This discrepancy could relate to differences in the behavioral paradigms. In our task, the animals likely used the visual location of a dot to properly time their movements. In other tasks, subjects were not necessarily seeking to time their actions so precisely and were not typically using a moving visual stimulus for behavioral guidance. Interestingly, however, in the one study that shared our operational definition for self-timed actions, based on movement-time distributions, a very similar response dynamic was observed (Lee and Assad, 2003). Moreover, in Lee and Assad's task, the animals did not use a visual aid for timing actions, suggesting that the observed modulation generalizes beyond visually guided movements.

Area 5 neurons are known to exhibit directionally selective modulations during delay periods of delayed hand movement tasks (Crammond and Kalaska, 1989). Preparatory activity of this sort is observed regularly in neurons of the motor system (Alexander and Crutcher, 1990). It is not clear whether one should consider the premovement modulations in our task as functionally equivalent or distinct from the delay-period modulations observed in other tasks. It is important to point out, however, that 
of the three blocks in our main task, it is the reaction block which most resembles a standard delayed-response task. In the reaction block, the animals knew what movement they were going to make, they just had to wait for a temporally delayed cue, a dot turn, to initiate that movement. Area 5 responses in the reaction block and, in fact, all blocks, could therefore include a tonic elevation (for burst cells) or suppression (for dip cells) related to the preparatory set. In this light, premovement firing-rate ramps specific to the active and active-delay modes could reflect a distinct modulation on top of the classical set-related activity. The dynamic of these ramps is consistent with a positive-feedback process that could play a role in the very last phases of initiating an action.

\section{References}

Alexander GE, Crutcher MD (1990) Preparation for movement: neural representations of intended direction in three motor areas of the monkey. J Neurophysiol 64:133-150.

Alexander GE, DeLong MR, Strick PL (1986) Parallel organization of functionally segregated circuits linking basal ganglia and cortex. Annu Rev Neurosci 9:357-381.

Burbaud P, Doegle C, Gross C, Bioulac B (1991) A quantitative study of neuronal discharge in areas 5,2 , and 4 of the monkey during fast arm movements. J Neurophysiol 66:429-443.

Commenges D, Seal J (1985) The analysis of neuronal discharge sequences: change-point estimation and comparison of variances. Stat Med 4:91-104.

Cook EP, Maunsell JH (2002) Dynamics of neuronal responses in macaque MT and VIP during motion detection. Nat Neurosci 5:985-994.

Crammond DJ, Kalaska JF (1989) Neuronal activity in primate parietal cortex area 5 varies with intended movement direction during an instructeddelay period. Exp Brain Res 76:458-462.

Deecke L (1996) Planning, preparation, execution, and imagery of volitional action. Brain Res Cogn Brain Res 3:59-64.

DiCarlo JJ, Maunsell JH (2004) Using neuronal latency to determine sensory-motor processing pathways in reaction time tasks. J Neurophysiol 93:2974-2986.

Efron B, Tibshirani RJ (1993) An introduction to the bootstrap. Boca Raton, FL: CRC.

Eskandar EN, Assad JA (2002) Distinct nature of directional signals among parietal cortical areas during visual guidance. J Neurophysiol 88:1777-1790.

Gemba H, Sasaki K (1984) Distribution of potentials preceding visually initiated and self-paced hand movements in various cortical areas of the monkey. Brain Res 306:207-214.

Gemba H, Matsuura-Nakao K, Matsuzaki R (2004) Preparative activities in posterior parietal cortex for self-paced movement in monkeys. Neurosci Lett 357:68-72.

Gold JI, Shadlen MN (2000) Representation of a perceptual decision in developing oculomotor commands. Nature 404:390-394.

Hanes DP, Schall JD (1996) Neural control of voluntary movement initiation. Science 274:427-430.

Hanes DP, Patterson II WF, Schall JD (1998) Role of frontal eye fields in countermanding saccades: visual, movement, and fixation activity. J Neurophysiol 79:817-834.

Hoover JE, Strick PL (1999) The organization of cerebellar and basal ganglia outputs to primary motor cortex as revealed by retrograde transneuronal transport of herpes simplex virus type 1. J Neurosci 19:1446-1463.
Johnson PB, Ferraina S, Bianchi L, Caminiti R (1996) Cortical networks for visual reaching: physiological and anatomical organization of frontal and parietal lobe arm regions. Cereb Cortex 6:102-119.

Kalaska JF, Caminiti R, Georgopoulos AP (1983) Cortical mechanisms related to the direction of two-dimensional arm movements: relations in parietal area 5 and comparison with motor cortex. Exp Brain Res 51:247-260.

Kelly RM, Strick PL (2003) Cerebellar loops with motor cortex and prefrontal cortex of a nonhuman primate. J Neurosci 23:8432-8444.

Lee IH, Assad JA (2003) Putaminal activity for simple reactions or selftimed movements. J Neurophysiol 89:2528-2537.

Marconi B, Genovesio A, Battaglia-Mayer A, Ferraina S, Squatrito S, Molinari M, Lacquaniti F, Caminiti R (2001) Eye-hand coordination during reaching. I. Anatomical relationships between parietal and frontal cortex. Cereb Cortex 11:513-527.

Matelli M, Govoni P, Galletti C, Kutz DF, Luppino G (1998) Superior area 6 afferents from the superior parietal lobule in the macaque monkey. J Comp Neurol 402:327-352.

Middleton FA, Strick PL (2000) Basal ganglia and cerebellar loops: motor and cognitive circuits. Brain Res Brain Res Rev 31:236-250.

Mink JW, Thach WT (1991) Basal ganglia motor control. I. Nonexclusive relation of pallidal discharge to five movement modes. J Neurophysiol 65:273-300.

Mountcastle VB, Lynch JC, Georgopoulos A, Sakata H, Acuna C (1975) Posterior parietal association cortex of the monkey: command functions for operations within extrapersonal space. J Neurophysiol 38:871-908.

Munoz DP, Wurtz RH (1993) Fixation cells in monkey superior colliculus. I. Characteristics of cell discharge. J Neurophysiol 70:559-575.

Okano K, Tanji J (1987) Neuronal activities in the primate motor fields of the agranular frontal cortex preceding visually triggered and self-paced movement. Exp Brain Res 66:155-166.

Prut Y, Fetz EE (1999) Primate spinal interneurons show pre-movement instructed delay activity. Nature 401:590-594.

Romo R, Schultz W (1992) Role of primate basal ganglia and frontal cortex in the internal generation of movements. III. Neuronal activity in the supplementary motor area. Exp Brain Res 91:396-407.

Romo R, Scarnati E, Schultz W (1992) Role of primate basal ganglia and frontal cortex in the internal generation of movements. II. Movementrelated activity in the anterior striatum. Exp Brain Res 91:385-395.

Sanger TD (2003) Childhood onset generalised dystonia can be modelled by increased gain in the indirect basal ganglia pathway. J Neurol Neurosurg Psychiatry 74:1509-1515.

Schultz W, Romo R (1992) Role of primate basal ganglia and frontal cortex in the internal generation of movements. I. Preparatory activity in the anterior striatum. Exp Brain Res 91:363-384.

Scudder CA, Kaneko CS, Fuchs AF (2002) The brainstem burst generator for saccadic eye movements: a modern synthesis. Exp Brain Res 142:439-462.

Seal J, Gross C, Bioulac B (1982) Activity of neurons in area 5 during a simple arm movement in monkeys before and after deafferentation of the trained limb. Brain Res 250:229-243.

Sirigu A, Daprati E, Ciancia S, Giraux P, Nighoghossian N, Posada A, Haggard $\mathrm{P}$ (2004) Altered awareness of voluntary action after damage to the parietal cortex. Nat Neurosci 7:80-84.

van Donkelaar P, Stein JF, Passingham RE, Miall RC (1999) Neuronal activity in the primate motor thalamus during visually triggered and internally generated limb movements. J Neurophysiol 82:934-945.

Yeterian EH, Pandya DN (1993) Striatal connections of the parietal association cortices in rhesus monkeys. J Comp Neurol 332:175-197. 